\title{
An Arterial Signal Coordination Optimization Model for Trams Based on Modified AM-BAND
}

\author{
Yangfan Zhou, ${ }^{1}$ Shunping Jia, ${ }^{1}$ Baohua Mao, ${ }^{1}$ Tin Kin Ho, ${ }^{2}$ and Wei Wei ${ }^{1}$ \\ ${ }^{1}$ MOE Key Laboratory for Urban Transportation Complex Systems Theory and Technology, School of Traffic and Transportation, \\ Beijing Jiaotong University, Beijing 100044, China \\ ${ }^{2}$ Shantou University, Guangdong 515063, China \\ Correspondence should be addressed to Shunping Jia; shpjia@bjtu.edu.cn
}

Received 5 March 2016; Revised 5 June 2016; Accepted 19 June 2016

Academic Editor: Filippo Cacace

Copyright (C) 2016 Yangfan Zhou et al. This is an open access article distributed under the Creative Commons Attribution License, which permits unrestricted use, distribution, and reproduction in any medium, provided the original work is properly cited.

\begin{abstract}
Modern trams are developing fast because of their characteristics like medium capability and energy saving. Exclusive way is always set in practice to avoid interruption from general vehicles, while trams have to stop at intersections frequently due to signal rules in the road network. Therefore, signal optimization has great effects on operational efficiency of trams system. In this paper, an arterial signal coordination optimization model is proposed for trams progression based on the Asymmetrical Multi-BAND (AMBAND) method. The AM-BAND is modified from the following aspects. Firstly, BAM-BAND is developed by supplementing active bandwidth constraints to AM-BAND. Assisted by the IBM ILOG CPLEX Optimization Studio, two arterial signals plans with eight intersections are achieved from AM-BAND and BAM-BAND for comparison. Secondly, based on the modified BAM-BAND, a BAM-TRAMBAND model is presented, which incorporates three constraints regarding tram operations, including dwell time at stations, active signal priority, and minimum bandwidth value. The case study and VISSIM simulation results show that travel times of trams decrease with signal plan from BAM-TRAMBAND comparing with the original signal plan. Moreover, traffic performance indicators such as stops and delay are improved significantly.
\end{abstract}

\section{Introduction}

Modern trams are medium capability urban public transit and develop fast in recent years of China for its characteristics of energy efficiency, environmental friendliness, and passenger comfort. As of 2015, trams are fully operated in nine cities with $180 \mathrm{~km}$ in total length which represents 5.1\% of overall urban rail transit distance in China [1]. Unlike other rail transit modes with exclusive right-of-way such as metro, light rail, and maglev, trams operate in an open road network and they are frequently interrupted by other vehicles and have to observe signal rules. To improve operational efficiency, two countermeasures are usually employed.

On one hand, exclusive way is provided for trams to avoid interweaving with other vehicles. On the other hand, signal priority strategies are adopted to ensure trams passing through intersections orderly and safely. Active, passive, and adaptive approaches are three main priority strategies [2]. Active priority provides early green, green extend, phase insertion, and other countermeasures to create extra green time for trams when they approach intersection [3, 4]. However, this method interferes with the original timing and imposes delays on other vehicles to certain extent. Sometimes even conducting early green or green extend strategies trams still probably arrive during red time. Besides active priority, green-wave band, as a passive priority strategy, is provided for trams in Athens [5]. Arterial signal coordination can create green-wave band. If a tram travels in green bandwidth, it will pass through all the arterial intersections without stop. This is more meaningful for trams with exclusive way, since there is no interruption from other vehicles. Therefore, it is necessary to explore signal coordination model for trams.

Morgan and Little [6] and Little [7] proposed an arterial signal coordination method for motorcade to maximize bandwidth. They converted the signal problem to a mixed integer linear programing problem to attain uniform cycle, phase time, offset, and progression speed. Solution procedure utilizing branch-and-bound algorithm was provided in the 


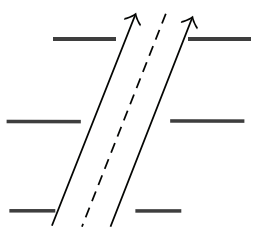

MAXBAND

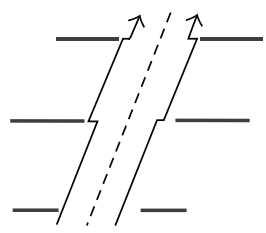

MULTIBAND

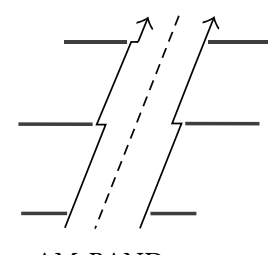

AM-BAND
FIgUre 1: Time-space diagram of MAXBAND, MULTIBAND, and AM-BAND.

study. In 1981, Little et al. [8] named this method MAXBAND and improved it by considering left-turn phase sequence and queue clearance time. Each band of the both-way green bands from MAXBAND owns the same width and is symmetrical along progression line. Having contracted by the Federal Highway Administration, the Texas Transportation Institute recognized that the left-turn phase sequence would be an important variable in multiarterial closed networks and proposed the MAXBAND-86 model [9]. Based on MAXBAND, Messer et al. [10], Chang and Messer [11], and Chaudhary and Messer [12] developed a series of PASSER programs to optimize arterial signal timing. In addition, Cohen [13] and Cohen and Mekemson [14] combined MAXBAND with TRANSYT (Traffic Network Study Tool). They used the bandwidth-based program MAXBAND to select proper leftturn phase sequence subsequently, followed by delay-based program TRANSYT to determine the final offsets. The resulting signal timing plan had lower delay than either class of programs individually.

For improving the utilization of green time, Gartner et al. $[15,16]$ relaxed the same band constraints along the progression line and presented a multiband method, which is the MULTIBAND model. Stamatiadis and Gartner [17] and Gartner and Stamatiadis [18] then realized its application in multiarterial traffic networks. Even though MULTIBAND relaxes the same band constraints, there is still a part of available band which cannot be utilized. In 2015, Zhang et al. [19] proposed an asymmetrical and multiband model named AM-BAND which relaxed the symmetrical constraints in MULTIBAND further. This model increases bandwidth and decreases the stops and delay of motorcades.

There is a gradual evolutionary relationship among MAXBAND, MULTIBAND, and AM-BAND. The schematic bandwidth results of the three models are shown in Figure 1. Although they widen the bandwidth, there is inactive area in the results. A certain band area is always left unused.

MAXBAND is designed for solving arterial signal coordination for general vehicles, but it is not entirely suitable for public transits because there is no time for stopping at stations in the bandwidth. Jeong and Kim [20, 21] modeled TRAMBAND by considering stops at stations for trams based on MAXBAND. Two both-way bandwidths for trams and general vehicles were achieved concurrently. Stops and delay at intersections for trams decreased from TRAMBAND compared with MAXBAND, while general vehicles delay increased slightly. Similarly, Dai et al. [22] introduced Model $X$ for bus green band, Model $Y$ for general vehicles band, and
Model $V$ for both green bands. Furthermore, Dai et al. [23] categorized intersections along the arterial according to the locations of bus stops. Models were then developed by optimizing the bandwidth for each group of intersections and connecting the central lines of green bands for adjacent groups. The both-way bandwidths for buses were obtained and bandwidths for different groups were not necessarily the same.

Most signal coordination methods for public transits are developed on the basis of MAXBAND, and they introduce constraints on stops at stations. These methods are clearly based on passive priority strategy. In fact, active priority and passive priority approaches are not in conflict. After a passive priority solution is confirmed, active priority is conducted for supplementing, which can enhance operation efficiency of trams and ensure smooth intersection passage without stop. Wang et al. [24] developed a double-level system for bus priority. The upper level was progression control. Cycle, green band, and offset were optimized according to the data of general vehicles from detectors. The lower level was bus priority control. Green extended and early start modes were adopted to achieve bus signal priority with respect to the upper level results for signal coordination. However, the existing arterial signal coordination model was only utilized but not optimized. Arterial signal coordination and actuated signal control are still two separate methods.

It should be specially mentioned that there are some researches that criticized classical traffic flow theories including green-wave theory. Kerner $[25,26]$ holds the view that green-wave breakdown occurred when the moving synchronized flow patterns propagated within the green-wave, and then the moving synchronized flow patterns transformed into a moving queue. It has to admit that three-phase traffic flow and queue problems indeed influence the effectiveness of MAXBAND in reality. However, trams' travelling characters and environment are different from general vehicles. On one hand, exclusive lane and its discrete arriving help to avoid trams' breakdown. On the other hand, compared with motorcade, it is easier to control the stopping, accelerating, decelerating, and speed of trams artificially or automatically according to the train diagram. Therefore, the modified model can be used better for trams.

Based on the literatures review above, an arterial signal coordination optimization model for trams is proposed by modifying AM-BAND in the following aspects:

(i) Active bandwidth: incorporate active bandwidth constraints.

(ii) Dwell time: incorporate constraints of trams' dwell time at station.

(iii) Active priority: provide early green and green extend signal priority strategies for trams at the intersection.

(iv) Minimum bandwidth: introduce minimum bandwidth value constraints to consider long tram body length.

The modified model with the active bandwidth constraint (i) is named BAM-BAND. The model with constraints (ii), 


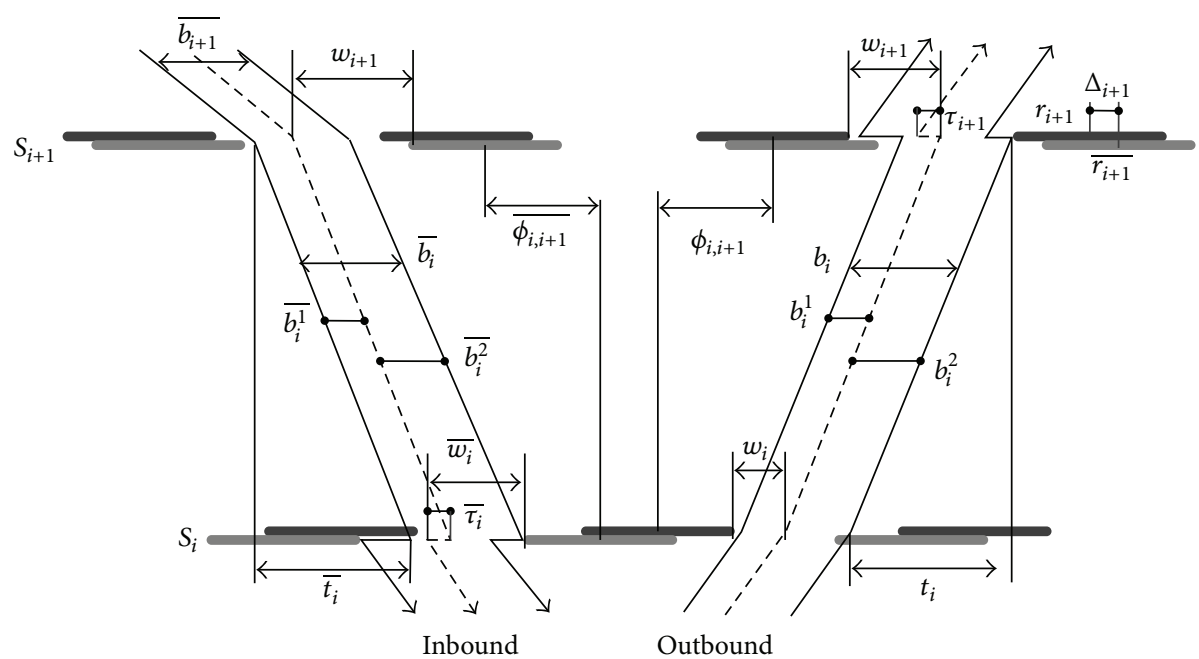

Figure 2: Time-space diagram of AM-BAND.

(iii), and (iv) based on BAM-BAND is denoted as BAMTRAMBAND. These two new models are still mixed integer linear programing problems. The remainder of this paper is organized as follows. Section 2 introduces the AM-BAND approach and discusses the results of an example. The bandwidth area is developed firstly in Section 3 and BAM-BAND is then proposed by introducing the active band bandwidth constraints. Finally in this section, the optimization model for trams, BAM-TRAMBAND, is proposed with constraints of dwell time, active priority, and minimum bandwidth. In Section 4, a case study and simulation analysis are conducted with the BAM-TRAMBAND approach and VISSIM software.

\section{AM-BAND Model and Application}

2.1. AM-BAND Model. AM-BAND model is an arterial signal coordination approach which falls in the category of passive priority. Signal timings of all intersections are fixed timing schemes. There are both green bands for two travel directions along the arterial including outbound and inbound as described in Figure 2.

The objective function of AM-BAND, as defined in (1), is to maximize weighted sum of bandwidths of outbound and inbound. Outbound bandwidth $b_{i}$ and inbound bandwidth $\bar{b}_{i}$ are composed of left and right parts, $b_{i}^{1}$ and $b_{i}^{2}$ and $\overline{b_{i}^{1}}$ and $\overline{b_{i}^{2}}$, which implies the green band of AM-BAND in each segment is asymmetrical and multiband. Constraints are (2) (11). The decision variables include $b_{i}^{1}, b_{i}^{2}, \overline{b_{i}^{1}}, \overline{b_{i}^{2}}, z, w_{i}, \overline{w_{i}}, t_{i}, \overline{t_{i}}$, $\delta_{i}, \overline{\delta_{i}}$, and $m_{i}$ :

$$
\max \quad B=\sum_{i=1}^{n-1}\left[a_{i}\left(b_{i}^{1}+b_{i}^{2}\right)+\overline{a_{i}}\left(\overline{b_{i}^{1}}+\overline{b_{i}^{2}}\right)\right]
$$

subject to $\left(1-k_{i}\right)\left(\overline{b_{i}^{1}}+\overline{b_{i}^{2}}\right)$

$$
\geq\left(1-k_{i}\right) k_{i}\left(b_{i}^{1}+b_{i}^{2}\right),
$$

$$
\begin{aligned}
& \frac{1}{C_{\max }} \leq z \leq \frac{1}{C_{\min }} \\
& b_{i}^{1} \leq w_{i} \leq\left(1-r_{i}\right)-b_{i}^{2} \\
& b_{i}^{1} \leq w_{i+1}+\tau_{i+1} \leq\left(1-r_{i+1}\right)-b_{i}^{2}, \\
& \overline{b_{i}^{2}} \leq \overline{w_{i}}-\overline{\tau_{i}} \leq\left(1-\overline{r_{i}}\right)-\overline{b_{i}^{1}}, \\
& \overline{b_{i}^{2}} \leq \overline{w_{i+1}} \leq\left(1-\overline{r_{i+1}}\right)-\overline{b_{i}^{1}}, \\
& \quad i=1,2, \ldots, n-1,
\end{aligned}
$$

$$
\begin{aligned}
& \left(\frac{1}{q}\right) b_{i}^{2} \leq b_{i}^{1} \leq q b_{i}^{2}, \\
& \left(\frac{1}{q}\right) \overline{b_{i}^{2}} \leq \overline{b_{i}^{1}} \leq q \overline{b_{i}^{2}},
\end{aligned}
$$

$$
+\left(r_{i}-r_{i+1}\right)-\left(\tau_{i+1}+\overline{\tau_{i}}\right)=m_{i},
$$$$
i=1,2, \ldots, n-1,
$$

$$
\left(\frac{d_{i}}{f_{i}}\right) z \leq t_{i} \leq\left(\frac{d_{i}}{e_{i}}\right) z
$$$$
\left(\frac{\overline{d_{i}}}{\overline{f_{i}}}\right) z \leq \overline{t_{i}} \leq\left(\frac{\overline{d_{i}}}{\overline{e_{i}}}\right) z,
$$

$$
i=1,2, \ldots, n-1,
$$




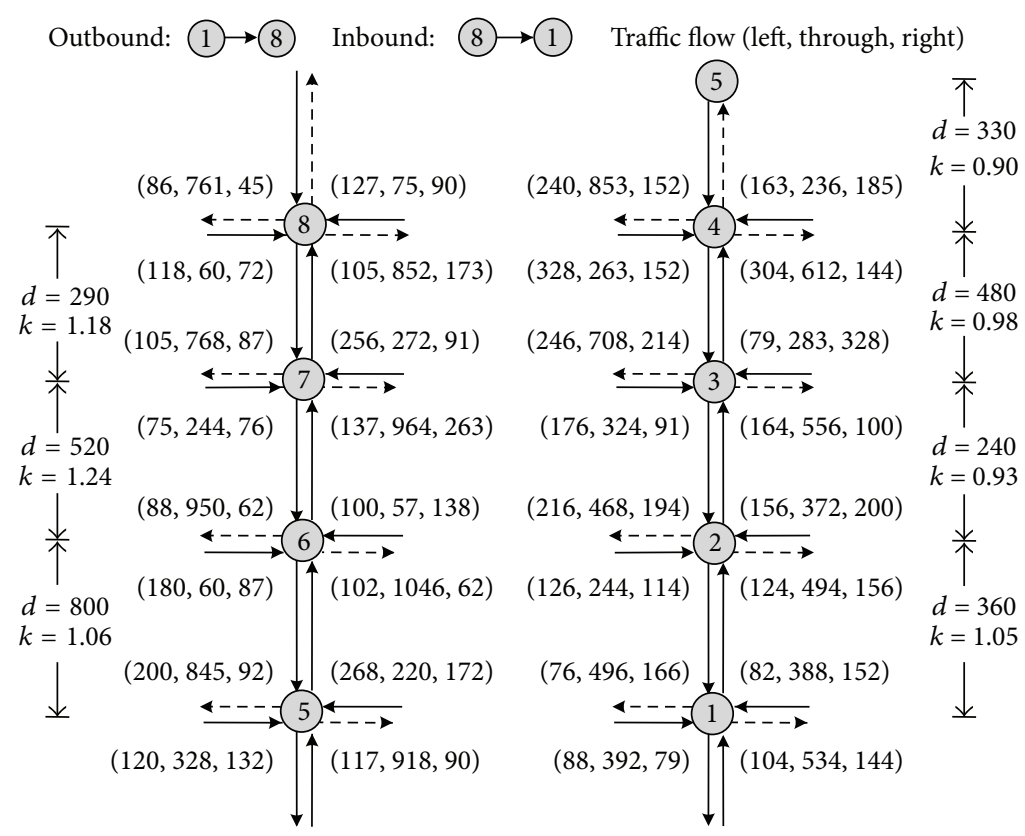

Figure 3: Data of the case study.

$$
\begin{array}{r}
\left(\frac{d_{i}}{h_{i}}\right) z \leq\left(\frac{d_{i}}{d_{i+1}}\right) t_{i+1}-t_{i} \leq\left(\frac{d_{i}}{g_{i}}\right) z, \\
\left(\frac{\overline{d_{i}}}{\overline{h_{i}}}\right) z \leq\left(\frac{\overline{d_{i}}}{\overline{d_{i+1}}}\right) \overline{t_{i+1}}-\overline{t_{i}} \leq\left(\frac{\overline{d_{i}}}{\overline{g_{i}}}\right) z, \\
i=1,2, \ldots, n-2,
\end{array}
$$

$m_{i}$ integer,

$\delta_{i}, \overline{\delta_{i}}$ zero/one variables,

$b_{i}^{1}, b_{i}^{2}, \overline{b_{i}^{1}}, \overline{b_{i}^{2}}, z, w_{i}, \overline{w_{i}}, t_{i}, \overline{t_{i}} \geq 0$.

Constraint (2) allows the arterial direction with a higher traffic volume to enjoy a wider progression band. Constraint (3) defines the lower and upper limits of cycle length. Two reciprocals are used here so that the remaining constraints can be expressed in linear form and solved by mixed integer linear programing. Constraint (4) is an interference constraint which ensures left and right boundaries do not interfere with the corresponding edges of red signals, respectively. Constraint (5) ensures that the left or right part of a progression band is nonzero. Constraint (6) is the well-known loop integer constraint. It releases the relationship among signal timing, bandwidth, and travelling speed. Values combinations of $\delta_{i}$ and $\overline{\delta_{i}}$ represent left-turn phase sequence. The optimal timing plan on straight and left-turn can be obtained from zero/one variables $\delta_{i}$ and $\overline{\delta_{i}}$ and left-turn green time $L_{i}\left(\overline{L_{i}}\right)$. Constraints $(7)$ and $(8)$ impose upper and lower limits on the travel speed of each segment and the speed change between consecutive segments. Left constraints (9), (10), and (11) are domains of definition.

The AM-BAND model consists of $23 n-19$ constraints and $11 n-6$ variables. For more details about AM-BAND model

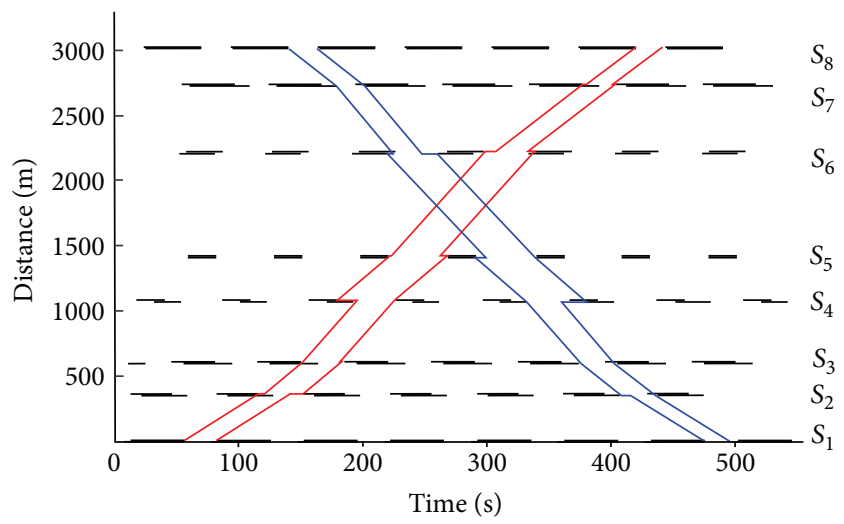

Figure 4: Time-space diagram of green band from AM-BAND.

see [19]. The notations and parameters used are explained in Notations. Some of their units are converted into cycles for mixed integer linear form.

2.2. Example Application for AM-BAND Model. For illustrating how it works and as the comparison results later, an arterial with eight intersections, as shown in Figure 3, is employed in this example. Lower and upper limits on cycle length are $70 \mathrm{~s}$ and $100 \mathrm{~s}$. Results from AM-BAND are illustrated in Table 1 . The optimization cycle is $70 \mathrm{~s}$, weighted sum of bandwidths is $426 \mathrm{~s}$, and average bandwidth is $30.4 \mathrm{~s}$. Timespace diagram of green band is given in Figure 4.

\section{Arterial Signal Coordination Optimization Model for Tram}

The optimization model is developed by modifying AMBAND in two steps. Firstly, the active bandwidth area is 
TABLE 1: Results from AM-BAND.

\begin{tabular}{|c|c|c|c|c|c|c|c|c|c|c|}
\hline$i$ & $b_{i}(\mathrm{~s})$ & $b_{i}^{1}(\mathrm{~s})$ & $b_{i}^{2}(\mathrm{~s})$ & $\overline{b_{i}}(\mathrm{~s})$ & $\overline{b_{i}^{1}}(\mathrm{~s})$ & $\overline{b_{i}^{2}}(\mathrm{~s})$ & $v_{i}(\mathrm{~m} / \mathrm{s})$ & $\overline{v_{i}}(\mathrm{~m} / \mathrm{s})$ & $\delta_{i}$ & $\overline{\delta_{i}}$ \\
\hline 1 & 26 & 15 & 11 & 20 & 11 & 9 & 6.0 & 6.0 & 1 & 1 \\
\hline 2 & 31 & 10 & 21 & 28 & 19 & 9 & 7.7 & 7.5 & 0 & 1 \\
\hline 3 & 30 & 10 & 20 & 28 & 19 & 9 & 11.0 & 11.0 & 0 & 1 \\
\hline 4 & 47 & 27 & 20 & 46 & 19 & 27 & 7.5 & 8.5 & 0 & 1 \\
\hline 5 & 39 & 26 & 13 & 40 & 13 & 27 & 10.7 & 10.2 & 0 & 0 \\
\hline 6 & 27 & 18 & 9 & 21 & 7 & 14 & 7.4 & 11.0 & 1 & 0 \\
\hline 7 & 22 & 15 & 7 & 21 & 7 & 14 & 7.3 & 7.5 & 0 & 1 \\
\hline 8 & & & & & & & & & 0 & 1 \\
\hline
\end{tabular}

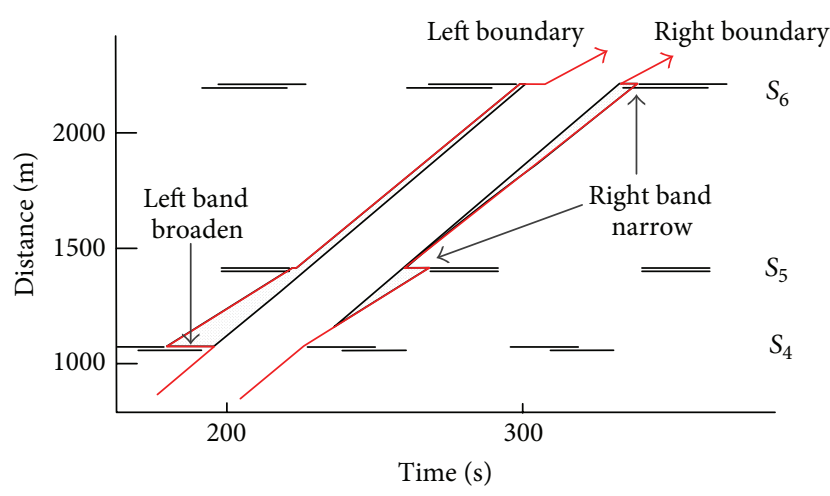

FIGURE 5: Inactive green band area.

defined and the BAM-BAND is constructed by supplementing active bandwidth constraints to AM-BAND. Secondly, with the consideration of dwell time, active priority, and minimum bandwidth constraints, an arterial signal coordination optimization model BAM-TRAMBAND for trams is developed.

3.1. Active Bandwidth Area. Although AM-BAND gives asymmetrical and multiband results to increase band's width, while not the entire bandwidth is active, it has been found that some of the bandwidth is always left unused. Because bandwidth is various, the bandwidth area as the relative availability can be indicated. In this study, bandwidth area $A_{0}$ refers to the sum of bandwidth values of each segment along the arterial multiplied by the matching distance between two intersections, as expressed in (12). The bandwidth area of the case in Section 2.2 from AM-BAND is $189880 \mathrm{~m} \cdot \mathrm{s}$; thus $A_{0}(\mathrm{AM}-\mathrm{BAND})=189880 \mathrm{~m} \cdot \mathrm{s}:$

$$
A_{0}=\sum_{i=1}^{n-1}\left[d_{i}\left(b_{i}^{1}+b_{i}^{2}\right)+\overline{d_{i}}\left(\overline{b_{i}^{1}}+\overline{b_{i}^{2}}\right)\right]
$$

The active bandwidth area $A_{1}$ is the part of $A_{0}$ which is available, while the inactive bandwidth area $A_{2}$ is the left part which is not available regardless of how the vehicles progress at reasonable speed. The inactive bandwidth area can be explained by intercepting the time-space diagram from $S_{4}$ to $S_{6}$ of green band. In Figure 5, if the left band in the outbound direction broadens, namely, $b_{i+1}^{1} \geq b_{i}^{1}$, there appears area which cannot be used even if the vehicle proceeds at maximum speed. Similarly, if the right band in outbound direction narrows, namely, $b_{i+1}^{2} \leq b_{i}^{2}$, once vehicle drives into the shadow area near to the right boundary, it will encounter red light at the next intersection even on full speed and overstep green band. Therefore, although bandwidth area from AM-BAND is $189880 \mathrm{~m} \cdot \mathrm{s}$, not the entire area is effective. Its active bandwidth area $A_{1}=35438 \mathrm{~m} \cdot \mathrm{s}$ and inactive bandwidth area $A_{2}=154442 \mathrm{~m} \cdot \mathrm{s}$. The active bandwidth area rate $\lambda=A_{2} / A_{0}=81 \%$, which indicates just $81 \%$ of the bandwidth area is available from AM-BAND for the case.

3.2. BAM-BAND Model. From the above discussion, there is inactive bandwidth area when left band broadens or right band narrows along the outbound direction. Similarly, if left band narrows or right band broadens along the inbound direction, inactive bandwidth area exists. Thus, active bandwidth constraints are necessary to eliminate invalid bandwidth. Active bandwidth constraints (13) beyond AM-BAND are introduced in the optimization model and thus the BAMBAND model:

$$
\begin{aligned}
b_{i+1}^{1} & \leq b_{i}^{1}, \\
b_{i+1}^{2} & \geq b_{i}^{2}, \\
\overline{b_{i+1}^{1}} & \geq \overline{b_{i}^{1}}, \\
\overline{b_{i+1}^{2}} & \leq \overline{b_{i}^{2}}, \\
& \quad i=1,2, \ldots, n-2 .
\end{aligned}
$$

The case study in Section 2.2 is then solved by BAMBAND and results from these two models are listed in Table 2. The optimization cycle is $75 \mathrm{~s}$. Weighted sum of bandwidths from BAM-BAND decreases by $7 \%$ and bandwidth area drops by $8 \%$ compared with AM-BAND, while active bandwidth area increases by $13 \%$. For the purpose of comparison, the cycle time is fixed at $70 \mathrm{~s}$. In this condition, weighted sum of bandwidths from BAM-BAND reduces by $14 \%$, and bandwidth area drops $15 \%$, while active bandwidth area improves $5 \%$. In addition, travel times of outbound and inbound save $9 \%$ and $14 \%$, respectively. These results prove that even though calculated bandwidth is smaller derived from BAM-BAND, the available bandwidth is in fact larger. 
TABLE 2: Results contrast of AM-BAND and BAM-BAND.

\begin{tabular}{lccc}
\hline & AM-BAND & \multicolumn{2}{c}{ BAM-BAND } \\
\hline$C(\mathrm{~s})$ & 70 & 75 & 70 \\
$B(\mathrm{~s})$ & 426 & $395(-7 \%)$ & $367(-14 \%)$ \\
$\sum_{i}^{n-1} t_{i}(\mathrm{~s})$ & 363 & $371(+2 \%)$ & $330(-9 \%)$ \\
$\sum_{i}^{n-1} \overline{t_{i}}(\mathrm{~s})$ & 339 & $290(-14 \%)$ & $293(-14 \%)$ \\
$A_{0}(\mathrm{~m} \cdot \mathrm{s})$ & 189880 & $173850(-8 \%)$ & $161450(-15 \%)$ \\
$A_{2}(\mathrm{~m} \cdot \mathrm{s})$ & 154442 & $173850(+13 \%)$ & $161450(+5 \%)$ \\
$\lambda$ & $81 \%$ & $100 \%$ & $100 \%$ \\
\hline
\end{tabular}

3.3. BAM-TRAMBAND Model. To extend this arterial signal coordination optimization model to trams, another three constraints including dwell time, active priority, and minimum bandwidth are introduced.

(1) Dwell Time Constraints. Dwell time constraints are

$$
\begin{aligned}
\min \Delta t_{i} & \leq \Delta t_{i} \leq \max \Delta t_{i}, \\
\min \overline{\Delta t_{i}} & \leq \overline{\Delta t_{i}} \leq \max \overline{\Delta t_{i}}, \\
& \quad i=1,2, \ldots, n-1,
\end{aligned}
$$

where $\Delta t_{i}\left(\overline{\Delta t_{i}}\right)$ denotes dwell time of outbound (inbound); $\min \Delta t_{i}\left(\min \overline{\Delta t_{i}}\right)$ denotes the minimum dwell time of outbound (inbound) ensuring boarding and alighting time of passengers; $\max \Delta t_{i}\left(\max \overline{\Delta t_{i}}\right)$ denotes the maximum dwell time of outbound (inbound) allowed. Dwell time is usually determined according to passenger numbers boarding and alighting at each station [27]. Moreover, travel speed and speed change constraints (15) and (16) replace (8) and (9), where $\varepsilon_{i}\left(\overline{\varepsilon_{i}}\right)$ is zero/one variable. If there is a station in segment $i$, it equals 1 . Otherwise, it equals 0 . Consider

$$
\begin{aligned}
\left(\frac{d_{i}}{f_{i}}\right) z & \leq t_{i}-\varepsilon_{i} \Delta t_{i} z \leq\left(\frac{d_{i}}{e_{i}}\right) z, \\
\left(\frac{\overline{d_{i}}}{\overline{f_{i}}}\right) z & \leq \overline{t_{i}}-\overline{\varepsilon_{i}} \overline{\Delta t_{i}} z \leq\left(\frac{\overline{d_{i}}}{\overline{e_{i}}}\right) z, \\
\left(\frac{d_{i}}{h_{i}}\right) z & \leq\left(\frac{d_{i}}{d_{i+1}}\right)\left(t_{i+1}-\varepsilon_{i+1} \Delta t_{i+1} z\right)-\left(t_{i}-\varepsilon_{i} \Delta t_{i} z\right) \\
& \leq\left(\frac{d_{i}}{g_{i}}\right) z, \\
\left(\overline{d_{i}}\right. & =\left(\frac{\overline{d_{i}}}{\overline{h_{i}}}\right) z\left(\overline{t_{i+1}}-\overline{\varepsilon_{i+1}} \overline{\Delta t_{i+1}} z\right)-\left(\overline{t_{i}}-\overline{\varepsilon_{i}} \overline{\Delta t_{i}} z\right) \\
& \leq\left(\frac{\overline{d_{i}}}{\overline{g_{i}}}\right) z, \quad i=1,2, \ldots, n-2 .
\end{aligned}
$$

(2) Active Priority Strategies. Arterial signal coordination is a passive priority approach. All signal timings are fixed. As the wave of travelling speed and deviation of dwell time at stations, trams are likely to miss the green band. It is possible to combine the advantages from active priority strategies, like early green and green extend, and that from passive priority.

Constraint (17) is adopted to replace (4). $\Delta r_{i \max }\left(\overline{\Delta r_{i \max }}\right)$ denotes the maximum early green time and $\Delta g_{i \max }\left(\overline{\Delta g_{i \max }}\right)$ denotes the maximum green extend time for trams. These two values should not be too large to avoid causing excessive delay to vehicles in other phases [28]. Consider

$$
\begin{aligned}
b_{i}^{1} & \leq w_{i} \leq 1-\left(r_{i}-\Delta r_{i \max } z-\Delta g_{i \max } z\right)-b_{i}^{2}, \\
b_{i}^{1} & \leq w_{i+1}+\tau_{i+1} \\
& \leq 1-\left(r_{i+1}-\Delta r_{(i+1) \max } z-\Delta g_{(i+1) \max } z\right)-b_{i}^{2}, \\
\overline{b_{i}^{2}} & \leq \overline{w_{i}}-\overline{\tau_{i}} \leq 1-\left(\overline{r_{i}}-\overline{\Delta r_{i \max }} z-\overline{\Delta g_{i \max }} z\right)-\overline{b_{i}^{1}}, \\
\overline{b_{i}^{2}} & \leq \overline{w_{i+1}} \\
& \leq 1-\left(\overline{r_{i+1}}-\overline{\Delta r_{(i+1) \max }} z-\overline{\left.\Delta g_{(i+1) \max } z\right)-\overline{b_{i}^{1}},}\right. \\
& i=1,2, \ldots, n-1 .
\end{aligned}
$$

(3) Minimum Bandwidth Constraints. The tram body length is quite long, ranges from $20 \mathrm{~m}$ to $50 \mathrm{~m}$. The bandwidth should be sufficiently wide for passing of the whole body. Objective function of optimization model is to maximize weighted sum of bandwidths, but there is a lack of requirement for bandwidth in each segment. As a consequence, bandwidth in some segments may be very small even if the objective function value is maximized. Constraint (18) is then required to ensure the minimum bandwidth. The minimum bandwidth value can be determined according to tram body length:

$$
\begin{aligned}
& b_{i}^{1}+b_{i}^{2} \geq b_{\min } z, \\
& \overline{b_{i}^{1}}+\overline{b_{i}^{2}} \geq b_{\min } z, \\
& \quad i=1,2, \ldots, n-1 .
\end{aligned}
$$

BAM-TRAMBAND is now completed and it is still a mixed integer linear programing problem. Constraints are (2), (3), (5), (6), (9) (11), and (13) (18). There are $31 n-31$ constraints and $13 n-8$ variables in the model.

\section{Case Study and Simulation Analysis}

The example in Section 2.2 is further analyzed in this case study. Four tram stations are set between intersections 1 and 2, 3 and 4, 5 and 6, and 6 and 7. And there is exclusive way for trams. The problem is modeled by BAM-TRAMBAND and the solution is calculated by IBM ILOG CPLEX Optimization Studio.

The optimization cycle is $80 \mathrm{~s}$, weighted sum of bandwidths is $445 \mathrm{~s}$, and average bandwidth is $31.8 \mathrm{~s}$. Other results are given in Table 3. Time-space diagram of green band is shown in Figure 6. 


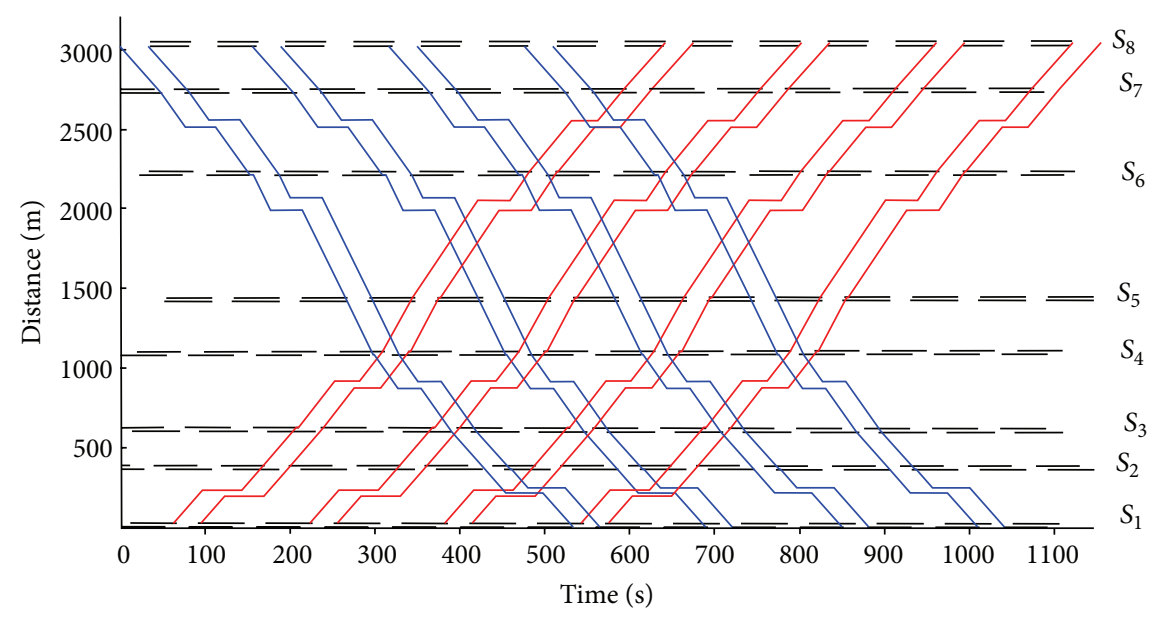

Figure 6: Time-space diagrams of green band from BAM-TRAMBAND.

TABLE 3: Results from BAM-TRAMBAND.

\begin{tabular}{|c|c|c|c|c|c|c|c|c|c|}
\hline \multirow{2}{*}{$i$} & \multicolumn{3}{|c|}{ Outbound } & \multicolumn{3}{|c|}{ Inbound } & \multirow{2}{*}{$\delta_{i}$} & \multirow{2}{*}{$\overline{\delta_{i}}$} & \multirow{2}{*}{ Absolute offset (s) } \\
\hline & $b_{i}(\mathrm{~s})$ & $t_{i}(\mathrm{~s})$ & $\Delta t_{i}(\mathrm{~s})$ & $\bar{b}_{i}(\mathrm{~s})$ & $\overline{t_{i}}(\mathrm{~s})$ & $\overline{\Delta t_{i}}(\mathrm{~s})$ & & & \\
\hline 1 & 33 & 106 & 46 & 31 & 103 & 43 & 1 & 0 & 0 \\
\hline 2 & 32 & 39 & - & 29 & 40 & - & 0 & 1 & 28 \\
\hline 3 & 30 & 100 & 31 & 29 & 92 & 28 & 0 & 1 & 65 \\
\hline 4 & 32 & 34 & - & 30 & 30 & - & 1 & 0 & 8 \\
\hline 5 & 33 & 138 & 38 & 30 & 111 & 38 & 1 & 1 & 42 \\
\hline 6 & 34 & 111 & 31 & 34 & 104 & 36 & 1 & 0 & 20 \\
\hline 7 & 34 & 48 & - & 34 & 48 & - & 0 & 1 & 51 \\
\hline 8 & & & & & & & 0 & 0 & 17 \\
\hline
\end{tabular}

An arterial with eight intersections and four stations according to the case is constructed in VISSIM simulation platform. The original timing plan and signal coordination optimization plan are simulated, respectively, with the period of 6 hours. The results under these two timing plans are obtained for comparison. Relevant results of general vehicles and trams are listed in Table 4.

Table 4 shows that, with the arterial signal coordination optimization plan from BAM-TRAMBAND, $12 \%$ and $20 \%$ of travel times of trams in two directions are saved, respectively, and standard deviations decrease almost by half. Under the original timing plan, trams stop more than 4 times at intersections, which indicates they meet red light at half of the intersections. The signal delay reaches $100 \mathrm{~s}$ in total. Under the optimization timing plan, trams pass through all these eight intersections without stop. Therefore, BAMTRAMBAND is effective for arterial signal coordination optimization and enhancing operation efficiency of trams.

Stop frequency and delay of general vehicles reduce slightly. This is because arterial signal coordination method only arranges phase sequences instead of changing the green time. Original timing cycle of each intersection ranges from $70 \mathrm{~s}$ to $100 \mathrm{~s}$. The optimization cycle is $80 \mathrm{~s}$. For those unsaturated intersections whose cycle lengths are longer than $80 \mathrm{~s}$ before optimization, the optimization timing decreases their cycle time. This is helpful to reduce signal delay to some degree.

\section{Conclusions}

In this paper, an arterial signal coordination optimization model is proposed for trams based on the AM-BAND method. The modified method makes two main improvements. The BAM-BAND and BAM-TRAMBAND models are proposed accordingly.

AM-BAND is an asymmetrical and multiband method. It relaxes the symmetrical progression band requirement in the well-known MULTIBAND model. The bandwidth is allowed to be larger in AM-BAND, because it is not necessarily symmetrical with respect to the progression line. It is found in this study that if the left band broadens or the right band narrows along outbound direction or the left band narrows or the right band broadens along inbound direction, a significant inactive bandwidth area may be exist.

BAM-BAND is developed by incorporating active bandwidth constraints into AM-BAND. It prevents the left band from broadening and the right band from narrowing along the outbound direction and the left band from narrowing and the right band from broadening along the inbound direction. Results obtained from BAM-BAND indicate fully 
TABLE 4: Simulation results of original timing plan and optimization timing plan.

\begin{tabular}{|c|c|c|c|c|}
\hline & & & Original timing & Optimization timing \\
\hline \multirow{4}{*}{ Travel time of tram (s) } & Mean value & Outbound & 702.42 & $619.88(-12 \%)$ \\
\hline & Standard deviation & Outbound & 55.84 & 22.27 \\
\hline & Mean value & Inbound & 696.01 & $553.75(-20 \%)$ \\
\hline & Standard deviation & Inbound & 29.00 & 15.18 \\
\hline \multirow{2}{*}{\multicolumn{2}{|c|}{ Average stop times at intersections of tram }} & Outbound & 4.54 & 0 \\
\hline & & Inbound & 4.30 & 0 \\
\hline \multirow{2}{*}{\multicolumn{2}{|c|}{ Average delay at intersections of tram (s) }} & Outbound & 100.79 & 0 \\
\hline & & Inbound & 97.81 & 0 \\
\hline \multicolumn{3}{|c|}{ Average stop times of general vehicles } & 1.79 & 1.69 \\
\hline \multicolumn{3}{|c|}{ Average stop delay of general vehicles (s) } & 44.56 & 39.58 \\
\hline
\end{tabular}

active bandwidth. Even though bandwidth reduces $15 \%$ from BAM-BAND compared with AM-BAND under the same conditions, active bandwidth area increases by $5 \%$. Available bandwidth area for vehicle becomes larger with the modified model.

There are stops at stations for trams. Active priority strategies can be adopted to ensure trams progression without stop at intersections, in addition to passive priority approach. Minimum bandwidth is necessary for trams because of long body length. BAM-TRAMBAND is then proposed by adding dwell time, active priority, and minimum bandwidth constraints to BAM-BAND. With BAM-TRAMBAND, travel times of trams in two directions are reduced by $12 \%$ and $20 \%$, respectively, compared with the original timing plan. Stops and delay at intersections drop to zero. BAM-TRAMBAND is able to develop efficient priority and improve service quality.

In the future, researches can be conducted from several aspects. Firstly, the signal coordination model discussed in this paper is only for one arterial. So, signal timings in the tram networks with interlaced lines are also worthy of studying. Secondly, one of trams' peculiarities is the limitation of timetable. To respect the operation schedule, timetable should be also considered when making signal coordination plans. Lastly, trams progression model is being developed to consider the influence of station's location such as near-side, far-side, and mid-block.

\section{Notations}

Sets

$S_{i}$ : Set of intersections.

Indices

$n$ : Number of intersections in the arterial

$i$ : Index of intersection and segment, $i=1,2, \ldots, n$.

\section{Parameters}

$a_{i}\left(\overline{a_{i}}\right)$ : Link specific weights in the two directions, where $a_{i}=\left(V_{i} / S V_{i}\right)^{p}$ and $\overline{a_{i}}=\left(\overline{V_{i}} / \overline{S V_{i}}\right)^{p}$
$V_{i}\left(\overline{V_{i}}\right): \quad$ Outbound (inbound) total directional volume (pcu/h)

$S V_{i}\left(\overline{S V_{i}}\right): \quad$ Outbound (inbound) saturation flow (pcu/h)

p: $\quad$ Exponential power whose values are $0,1,2$, and 4 usually used

$k_{i}$ : $\quad$ Target ratios of inbound to outbound bandwidth on section $i$; if $k_{i}=1, b_{i}=\bar{b}_{i}$

$C_{\max }$ and $C_{\min }$ : Upper and lower limits on cycle length (s)

$\tau_{i}\left(\overline{\tau_{i}}\right): \quad$ Outbound (inbound) queue clearance time at $S_{i}$ (cycles)

$r_{i}\left(\overline{r_{i}}\right): \quad$ Outbound (inbound) red time at $S_{i}$ (cycles)

$q$ : $\quad$ Can be any positive real number (e.g., $1,2,3, \ldots)$

$L_{i}\left(\overline{L_{i}}\right): \quad$ Time allocated for outbound (inbound)

left-turn green at $S_{i}$ (cycles)

$G_{i}\left(\overline{G_{i}}\right): \quad$ Outbound (inbound) green time for

through traffic at $S_{i}$ (cycles)

$d_{i}\left(\overline{d_{i}}\right): \quad$ Distance between $S_{i}$ and $S_{i+1}$ outbound (inbound) (m)

$e_{i}, f_{i}\left(\overline{e_{i}}, \overline{f_{i}}\right): \quad$ Lower/upper limits on outbound (inbound) speed $(\mathrm{m} / \mathrm{s})$

$h_{i}, g_{i}\left(\overline{h_{i}}, \overline{g_{i}}\right): \quad$ Lower/upper limits on change in outbound (inbound) speed $(\mathrm{m} / \mathrm{s})$.

\section{Intermediate Variables}

$B: \quad$ Weighted sum of bandwidth of outbound and inbound; average of it is $B^{\prime}=B / 2(n-1)$ (cycles)

$v_{i}\left(\overline{v_{i}}\right): \quad$ Travel speed of outbound from $S_{i}$ to $S_{i+1}$, travel speed of inbound from $S_{i+1}$ to $S_{i}(\mathrm{~m} / \mathrm{s})$

$\phi_{i, i+1}\left(\overline{\phi_{i, i+1}}\right):$ Internode offsets (cycles)

$\Delta_{i}: \quad$ Intranode offset, which is the time difference between the center of $r_{i}$ and the nearest center of $\overline{r_{i}}$; it is positive if the center of $r_{i}$ is to the right of the center of $\overline{r_{i}}$ (cycles). 


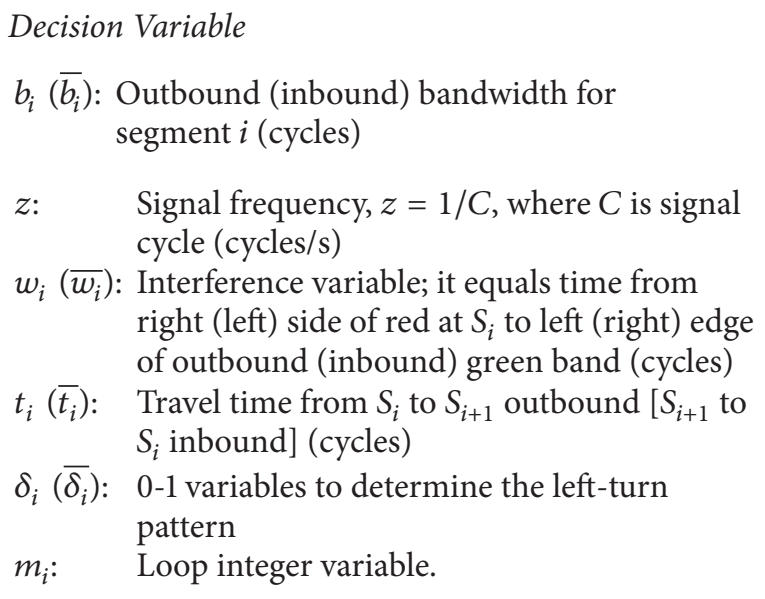

\section{Competing Interests}

The authors declare that there are no competing interests regarding the publication of this paper.

\section{Acknowledgments}

This research was funded by the Fundamental Research Funds for the Central Universities (2015YJS090), the National Basic Research Program of China (no. 2012CB725406), and the National Natural Science Foundation of China (NSFC) (no. 71390332).

\section{References}

[1] China Association of Metro, 2015 Annual Statistical Analysis Report of Urban Rail Transit, China Association of Metro, Beijing, China, 2016.

[2] J. Chang, An Overview of Transit Signal Priority, ITS America, Washington, DC, USA, 2002.

[3] M. A. C. Aleman, Evaluation of Bus Priority Strategies for BRT Operations, Massachusetts Institute of Technology, Boston, Mass, USA, 2013.

[4] Z. S. Yang, C. L. Tian, Y. Yu, and X. W. Li, “The BRT signal priority algorithm based on RFID," in Proceedings of the $3 \mathrm{rd}$ International Conference on Computer Science and Network Technology (ICCSNT '13), pp. 561-564, Dalian, China, October 2013.

[5] D. Sermpis, P. Papadakos, and K. Fousekis, "Tram priority at signal-controlled junctions," Proceedings of the Institution of Civil Engineers-Transport, vol. 165, no. 2, pp. 87-96, 2012.

[6] J. T. Morgan and J. D. C. Little, "Synchronizing traffic signals for maximal bandwidth," Operations Research, vol. 12, no. 6, pp. 896-912, 1964.

[7] J. D. C. Little, "The synchronization of traffic signals by mixedinteger linear programming," Operations Research, vol. 14, no. 4, pp. 568-594, 1966.

[8] J. D. C. Little, M. C. Kelson, and N. H. Gartner, MAXBAND: A Versatile Program for Setting Signals on Arteries and Triangular Networks, Alfred P. Sloan School of Management, Massachusetts Institute of Technology, Cambridge, Mass, USA, 1981.

[9] E. C.-P. Chang, S. L. Cohen, C. Liu, N. A. Chaudhary, and C. Messer, "MAXBAND-86: program for optimizing left-turn phase sequence in multiarterial closed networks," Transportation Research Record, no. 1181, pp. 61-67, 1988.

[10] C. J. Messer, R. H. Whitson, C. L. Dudek, and E. J. Romano, "A variable-sequence multiphase progression optimization program," Transportation Research Record, vol. 445, pp. 24-33, 1973.

[11] E. C. P. Chang and C. J. Messer, Arterial Signal Timing Optimization Using PASSER II-90-Program User's Manual, U.S. Department of Transportation, 1991.

[12] N. A. Chaudhary and C. J. Messer, PASSER IV-96, Version 2.1, User/Reference Manual, US Department of Transportation, 1996.

[13] S. L. Cohen, "Concurrent use of MAXBAND and TRANSYT signal timing programs for arterial signal optimization," Transportation Research Record, no. 906, pp. 81-84, 1983.

[14] S. L. Cohen and J. R. Mekemson, "Optimization of left-turn phase sequence on signalized arterials," Transportation Research Record, no. 1021, pp. 53-58, 1985.

[15] N. H. Gartner, S. F. Assmann, F. Lasaga, and D. L. Hous, "MULTIBAND—a variable-bandwidth arterial progression scheme," Transportation Research Record, no. 1287, pp. 212-222, 1990.

[16] N. H. Gartner, S. F. Assman, F. Lasaga, and D. L. Hou, "A multi-band approach to arterial traffic signal optimization," Transportation Research Part B, vol. 25, no. 1, pp. 55-74, 1991.

[17] C. Stamatiadis and N. H. Gartner, "MULTIBAND-96: a program for variable-bandwidth progression optimization of multiarterial traffic networks," Transportation Research Record, no. 1554, pp. 9-17, 1996.

[18] N. H. Gartner and C. Stamatiadis, "Arterial-based control of traffic flow in urban grid networks," Mathematical and Computer Modelling, vol. 35, no. 5-6, pp. 657-671, 2002.

[19] C. Zhang, Y. Xie, N. H. Gartner, C. Stamatiadis, and T. Arsava, "AM-band: an asymmetrical multi-band model for arterial traffic signal coordination," Transportation Research, Part C: Emerging Technologies, vol. 58, pp. 515-531, 2015.

[20] Y. Jeong and Y. Kim, "Tram passive signal priority strategy based on the MAXBAND model," KSCE Journal of Civil Engineering, vol. 18, no. 5, pp. 1518-1527, 2014.

[21] Y. Jeong and Y. Kim, "Bus coordination model for passive signal priority," in Proceedings of the 9th International Conference of Eastern Asia Society for Transportation Studies, 2011.

[22] G. Y. Dai, H. Wang, and W. Wang, "A bandwidth approach to arterial signal optimisation with bus priority," Transportmetrica A: Transport Science, vol. 11, no. 7, pp. 579-602, 2015.

[23] G. Y. Dai, H. Wang, and W. Wang, "Signal optimization and coordination for bus progression based on MAXBAND," KSCE Journal of Civil Engineering, vol. 20, no. 2, pp. 890-898, 2016.

[24] D. H. Wang, H. Zhu, Y. M. Bie, and Q. Wei, "Bus signal priority method at arterial signal progression," Journal of Southeast University (Natural Science Edition), vol. 41, no. 4, pp. 859-865, 2011.

[25] B. S. Kerner, "Criticism of generally accepted fundamentals and methodologies of traffic and transportation theory: a brief review," Physica A: Statistical Mechanics and Its Applications, vol. 392, no. 21, pp. 5261-5282, 2013.

[26] B. S. Kerner, "Three-phase theory of city traffic: moving synchronized flow patterns in under-saturated city traffic at signals," Physica A: Statistical Mechanics and Its Applications, vol. 397, no. 1, pp. 76-110, 2014. 
[27] S. Chen, R. Zhou, Y. Zhou, and B. Mao, "Computation on bus delay at stops in Beijing through statistical analysis," Mathematical Problems in Engineering, vol. 2013, Article ID 745370, 9 pages, 2013.

[28] Y.-Y. Yang, B.-H. Mao, J.-P. Zhang, L.-P. Gao, and S.-K. Chen, "Research on signal priority simulation for bus rapid transit using cellular automaton model," China Journal of Highway and Transport, vol. 23, no. 5, pp. 90-95, 2010. 


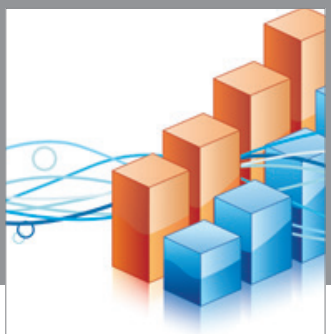

Advances in

Operations Research

vatem alat4

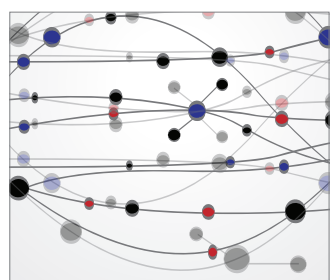

\section{The Scientific} World Journal
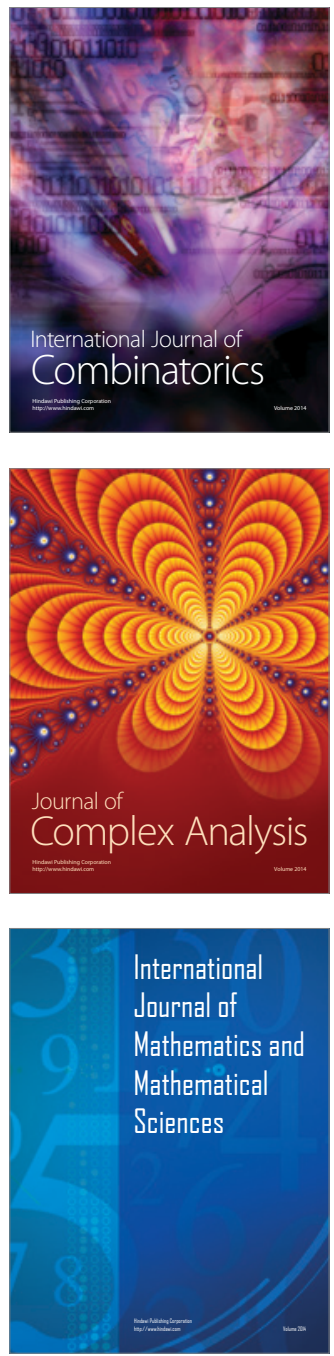
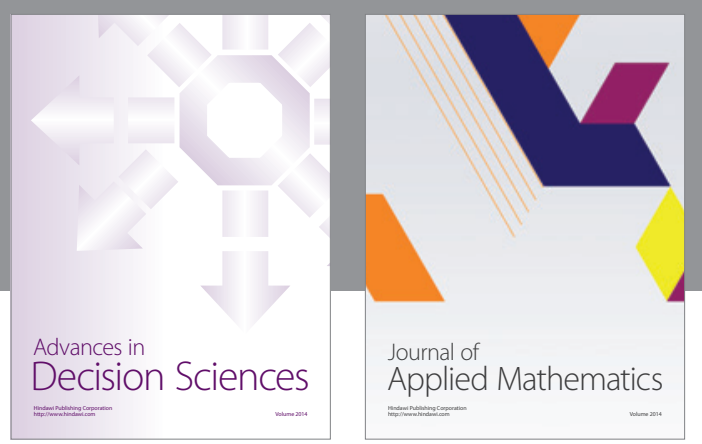

Algebra

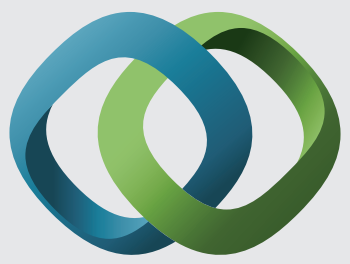

\section{Hindawi}

Submit your manuscripts at

http://www.hindawi.com
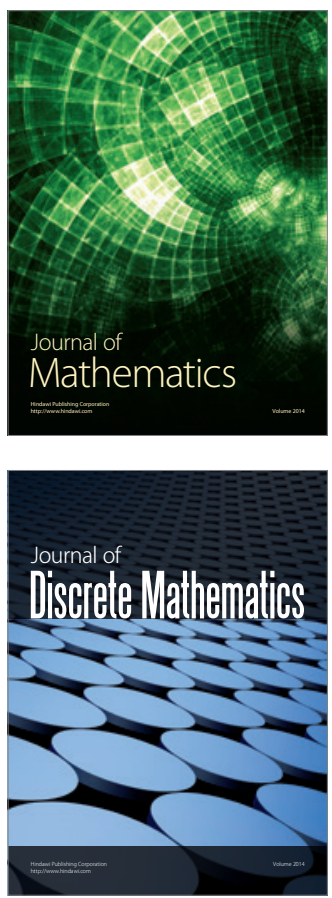

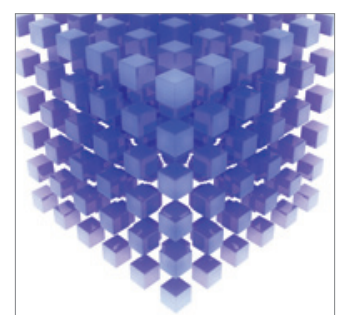

Mathematical Problems in Engineering
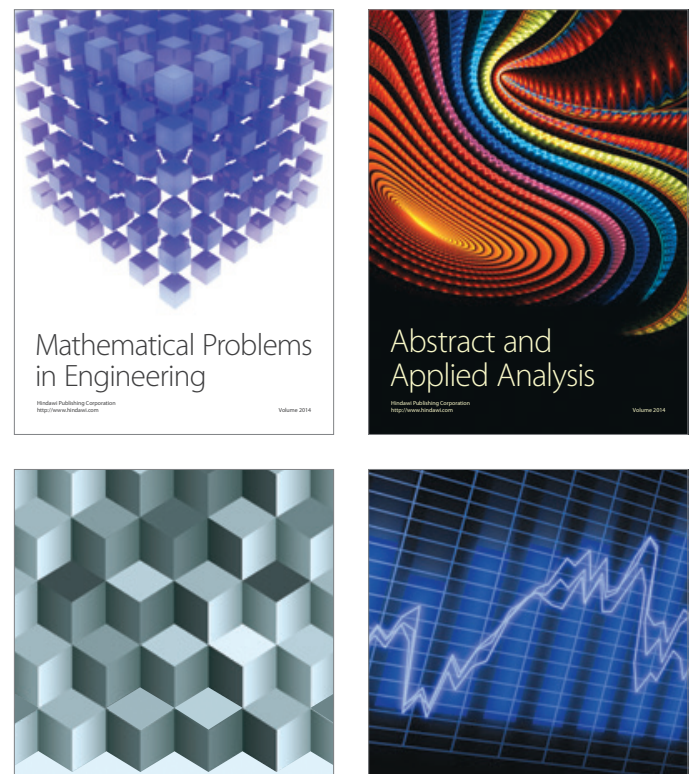

Journal of

Function Spaces

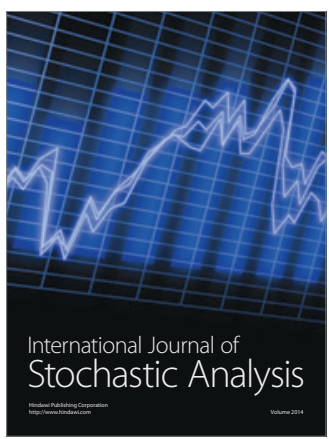

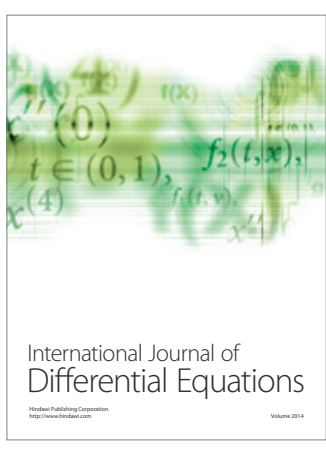
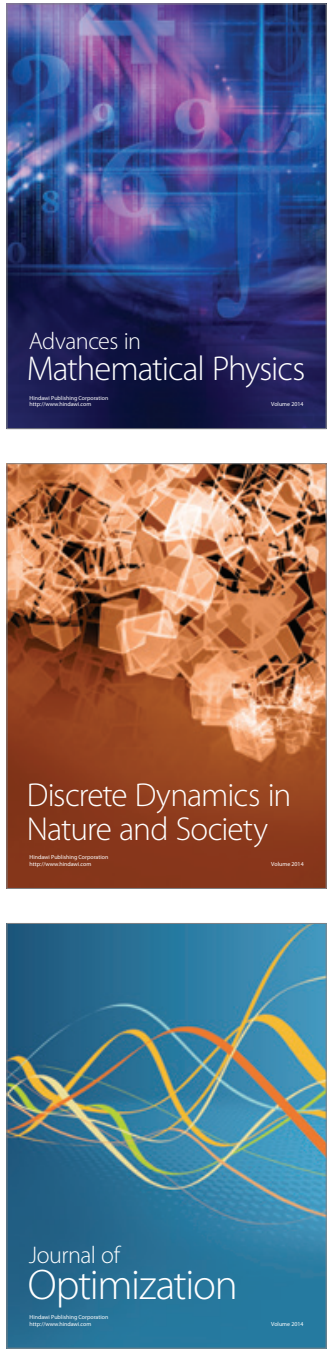